\title{
BMJ Open Construction of the National Brain Tumor Registry of China for better management and more efficient use of data: a protocol
}

\author{
Liwei Zhang, ${ }^{1}$ Wang Jia, ${ }^{1}$ Nan $\mathrm{Ji},{ }^{1}$ Deling Li (D) , ${ }^{1}$ Dan Xiao, ${ }^{2}$ Guang-Liang Shan, ${ }^{3}$ \\ Tao Wang, ${ }^{4}$ Xiong Xiao ${ }^{1}$
}

To cite: Zhang L, Jia W, Ji N, et al. Construction of the National Brain Tumor Registry of China for better management and more efficient use of data: a protocol. BMJ Open 2021;11:e040055. doi:10.1136/ bmjopen-2020-040055

- Prepublication history for this paper is available online. To view these files, please visit the journal online (http://dx.doi org/10.1136/bmjopen-2020040055).

Received 04 May 2020 Revised 01 August 2020 Accepted 09 October 2020

Check for updates

(C) Author(s) (or their employer(s)) 2021. Re-use permitted under CC BY-NC. No commercial re-use. See rights and permissions. Published by BMJ.

${ }^{1}$ Department of Neurosurgery, Beijing Tiantan Hospital, Beijing, China

${ }^{2}$ China National Clinical

Research Center for

Neurological Diseases, Beijing

Tiantan Hospital, Capital Medical University, Beijing, China

${ }^{3}$ Department of Epidemiology and Statistics, Institute of Basic Medical Sciences, Chinese Academy of Medical Sciences, School of Basic Medicine, Peking Union Medical College, Beijing, China

${ }^{4}$ Information Center, Beijing Tiantan Hospital, Beijing, China

Correspondence to

Dr Liwei Zhang;

Zhangliweittyy@163.com

\section{ABSTRACT}

Introduction Brain tumours encompass a complex group of intracranial tumours that mostly affect young adults and children, with a high incidence rate and poor prognosis. It remains impossible to systematically collect data on patients with brain tumours in China and difficult to perform in-depth analysis on the status of brain tumours, medical outcomes or other important medical issues through a multicentre clinical study. This study describes the first nation-wide data platform including the entire spectrum of brain tumour entities, which will allow better management and more efficient application of patient data in China.

Methods and analysis The National Brain Tumor Registry of China (NBTRC) is a registry of real-word clinical data on brain tumours. It is established and managed by the China National Clinical Research Center for Neurological Diseases and administered by its scientific and executive committees. The 54 participating hospitals of the NBTRC are located in 27 provinces/municipalities, performing more than 40000 brain tumour surgeries per year. The data consist of in-hospital medical records, images and follow-up information after discharge. Data can be uploaded in three ways: the web portal, remote physical servers and offline software. The data quality control scheme is seven-dimensional. Each participating hospital could focus on a single pathology subtype and public subtypes of brain tumour for which they expect to conduct related multicentre clinical research. The standardised workflow to conduct clinical research is based on the benefit-sharing mechanism. Data collection will be conducted continuously from 1 February 2019 to 31 January 2024.

Ethics and dissemination Informed consent will be obtained from all participants. Consent for the adolescents participation will be also obtained from their guardians via written consent. The results will be published in professional journals, in both Chinese and English. Trial registration number Chinese Clinical Trial Registry (ChiCTR1900021096).

\section{INTRODUCTION}

Necessity for a brain tumour registry system

Brain tumours consist of a complex group of intracranial tumours that mostly affect young
Strengths and limitations of this study

- The National Brain Tumor Registry of China (NBTRC) is the first nation-wide data platform that includes the entire spectrum of brain tumour entities in China.

- The NBTRC provides reliable macro-policy-making reference for the government authority, assists health professionals manage clinical data, helps patients obtain access to high-quality and convenient medical services, and enables researchers conduct nation-wide multicentre clinical research.

- The NBTRC has the standardised and prespecified follow-up interval and automatically provides data entry templates based on the pathology and location of the tumour, which will significantly improve the quality and comparability of follow-up data among participating hospitals and motivate each hospital to collect follow-up data.

- The NBTRC adopts three flexible ways for data collection, which will increase the inclusiveness of the NBTRC to collect data from as many hospitals as possible, improve the capability of the participating researchers and hospitals, and benefit them with this established process for accessing information.

- The data of the NBTRC are collected from hospitals rather than communities; thus, it is not a populationbased incidence registry, which may reduce the representativeness of data.

adults and children, with a high incidence rate and poor prognosis.

In the USA, tumours of the central nervous system, especially brain tumours, are the most common cause of cancer and cancer-related deaths in children aged $<15$ years and the third most common in people aged 15-39 years. ${ }^{1}$ In China, between 2012 and 2015, the 5-year survival rate of patients with brain tumours was only $26.7 \%$, which is much lower than the $40.5 \%$ average for all tumours. ${ }^{2}$

It is imperative to obtain the reliable data of brain tumours so as to make clear the epidemiological characteristics of brain 
tumours and provide high-quality data for the multicentre clinical study. However, the high-quality data for brain tumours, including the comprehensive statistical data, are not always available in many countries. China is no exception. There are three reasons for this dilemma. First, the classification of brain tumours is so complex, with more than 200 subtypes, ${ }^{3}$ that different doctors may not totally agree on the diagnosis of the same patient. Second, data elements, such as data structure, data type and index definition, are not unified and standardised among hospitals and study groups, which makes it difficult to pool data from multiple sources for cooperation among hospitals and study groups. Third, current cancer registration systems may miss the substantial portion of all brain tumours; their data are limited in scope, and long lag exists before the information becomes available.

To collect and use the medical data more efficiently, the National Institutes of Health and the National Cancer Institute proposed the urgent demand to build a 'cancer knowledge network'. ${ }^{4}$ This is really needed in the field of brain tumours as well. The basis of the multicentre clinical study on brain tumours includes accurate pathology diagnosis based on the most recognised criteria, unified and standardised data registry, and real-time data entry shortly after patient discharge.

\section{Brain tumour registries in the world}

Brain tumour registries are established in several developed countries to supplement clinical research for the prevention and treatment of brain tumours. For example, the Central Brain Tumor Registry of the United States (CBTRUS) is a population-based brain tumour registry, representing $100 \%$ of the nation's population. ${ }^{5}$ The UK launched a cancer registry that includes brain tumours, covering the nation's entire population. Countries with relatively smaller population, such as Japan and Finland, also have their own brain tumour registries. ${ }^{6-8}$

The brain tumour registries in these countries integrate a large amount of valuable data, with great benefits. The epidemiological distribution of the disease can be determined ${ }^{9-11}$ and changing trends in incidence can be monitored. ${ }^{712}$ The collection of data from multiple channels and the relatively large sample size allow the analysis of aetiological, influencing and predictive factors with great statistical significance. ${ }^{13-15}$ The internationalisation of the registry and integration with other disciplines can promote international cooperation and multidisciplinary development in brain tumour research. ${ }^{16}{ }^{17}$ Finally, by applying high-quality and reliable data from the registry, in-depth analysis will aid responses to social problems in the medical field and optimise the nation's healthcare system. ${ }^{18} 19$

It should be noted that the successful construction of the registry system urgently requires the support of the government and academic institutions, the active participation of neurosurgery centres and the formulation of reasonable rules and regulations. For most countries, these are formidable barriers to create such system for the registry of brain tumours. In addition, due to the high mobility of patients, few registry systems can collect information on the patient's entire disease process, especially postdischarge information such as recurrence, death and survival.

\section{Brain tumour registry in China and limitations}

China is the largest developing country without a nationwide brain tumour registry. It remains impossible to systematically collect data on patients with brain tumours in China and difficult to perform in-depth analysis on the status of brain tumours, medical outcomes and other important medical issues that affect the Chinese population through the multicentre clinical study.

In 2008, the National Central Cancer Registry (NCCR) of China was established, the mission of which is to collect data from local population-based cancer registries in local hospitals and community health centres, covering $16.43 \%$ of the nation's population until now. ${ }^{2021}$

According to the annual report from the NCCR, the incidence of brain tumours in the rural population is $6.41 / 10^{5}$, the ninth highest of all tumours in the rural population of China. ${ }^{22}$ This is significantly lower than $24.56 / 10^{5}$ reported by a cross-sectional survey in five regional centres in China ${ }^{23}$ and also lower than 21.42/10 reported by the brain tumour registry of the USA. ${ }^{5}$ Probably there are two reasons for these discrepancies. First, in addition to brain tumours, the NCCR is concerned about all types of malignant tumours, such as lung, liver and stomach. However, the benign brain tumour pathology entities are not included in that system. Second, as the calculation of these indicators is based on data collection done several years before, the diagnosis technology, data collection personnel skills and other aspects might need further improving at that time. Therefore, the data of a registry dedicated to the entire spectrum of brain tumours are urgently needed to resolve these problems in China.

Local hospitals and community health centres are often hampered by conditions that compromise the accurate diagnosis of brain tumours, such as incomplete imaging equipment, inconsistent diagnostic criteria and lack of experience. However, if the brain tumour registry system is located at large general hospitals where there are advanced neurosurgical skills, patient information will be collected more directly, accurately and comprehensively. Therefore, a professional nation-wide brain tumour registry system in China is necessary to collect accurate information from representative hospitals and regions, on patients with all pathological types of brain tumour.

\section{Importance of networking medical resources at participating hospitals}

Because of the unbalanced development of neurosurgical medical resources and lack of efficient medical referral ${ }^{24}$ and clinical data sharing mechanism in China, patients with brain tumours have difficulties in seeking medical service. In some large hospitals in China, patients with brain tumour have to wait several months for medical 
appointment. It is common to see patients and their relatives carrying heavy bundles of radiological films from hospital to hospital, with risk of loss or damage of hardcopy images.

Although this situation cannot be changed immediately, a brain tumour registry platform may provide the possibility to maximise the accessibility of medical resources of participating neurosurgery departments and facilitate faster medical referrals, and effective and efficient clinical examination result exchange among participating hospitals. This platform can also provide the theoretical foundation and practical experience for the development of a system to interconnect medical resources throughout the whole of China.

The development of data networks or data sharing system should focus not only on information technology needs but also on the complex regulatory, legal, security, privacy and workflow requirements to support the infrastructures so as to ensure the normal application of the system and achieve the expected consequence after the pilot stage. ${ }^{25}$ These are the issues that we should consider when establishing such brain tumour registry system in the nation-wide level. In this study, the organisation and management, participating hospitals, data collection methods, quality control scheme and benefit-sharing mechanism of the National Brain Tumor Registry of China (NBTRC) are described.

\section{METHODS AND ANALYSIS}

The NBTRC is the registry of real-word clinical data on brain tumours. It will collect, process and persist data, and provide data for the multicentre clinical studies of brain tumours and assist in the organisation and coordination of multicentre studies.

\section{Organisation and management of the NBTRC}

In January 2018, the NBTRC was established and managed by the China National Clinical Research Center for Neurological Diseases (NCRC-ND), which is merely the national centre authorised by Chinese government and organised by Beijing Tiantan Hospital. ${ }^{26}$ The NBTRC is chaired by Professor Liwei Zhang, the president of Chinese Neurosurgeons Association and vice president of Beijing Tiantan Hospital.

The NBTRC is administered by its scientific and executive committee. The scientific committee designs and guides to establish and operate the platform and assesses the scientific rationale and feasibility of research protocols. The executive committee is responsible for routine management and technical support.

\section{Inclusion criteria of participating hospitals}

National or regional neurosurgery centres in China participate in the NBTRC. The participating hospitals should meet all the following criteria. First, each hospital should perform at least 100 brain tumour operations annually and should be capable of histological and molecular diagnoses of pathologies for brain tumours. Second, each hospital should be equipped with systems for electronic medical records, standardised picture archiving and communication, and hospital information. Third, each hospital should cooperate with the benefitsharing mechanism and comply with the quality control standard of the NBTRC. Finally, each hospital should have enough well-trained clinical research assistants to upload data and perform follow-ups of postoperative patients.

\section{Characteristics of the participating hospitals}

As of April 2020, there are 54 hospitals that met the inclusion criteria for participation in the NBTRC, acknowledged the benefit-sharing mechanism, signed the participation contract and are now authorised as the first cluster of participating hospitals.

These 54 hospitals are located in 27 provinces/municipalities in China and evenly distributed across the country (figure 1). These hospitals accommodate 1653 neurosurgeons, with a cumulative annual average of 43584 brain tumour surgeries from 2012 to 2018.

\section{Patient enrolment criteria}

All the patients with primary brain tumours (malignant and non-malignant pathology entities) are enrolled in the NBTRC from the participating hospitals. The diagnosis is based on the 2016 WHO Classification of Tumors of the Central Nervous System, the newly recognised pathology guideline. The patients can be categorised into three types: (1) confirmed by postoperative pathology after operation; (2) pituitary adenoma that are diagnosed based on clinical and radiological features, and the patients require in-hospital treatment rather than operation; and (3) brainstem glioma, an extremely rare brain tumour, can be diagnosed only on radiology due to international diagnosis criteria.

\section{The collected information and indexes}

The data collected in the NBTRC consist of in-hospital medical records, patient images and follow-up information after discharge. The in-hospital information of each patient mainly includes demographics, clinical symptoms and signs, standardised pathology diagnosis, operation record and adjuvant therapy such as radiotherapy and chemotherapy. The imaging data, including different sequences of MRI, CT and positron emission tomography (when conducted), are in the Digital Imaging and Communications in Medicine format. The follow-up information mainly includes the treatment after discharge, the dynamic change of clinical symptoms and signs, and the patient's prognosis.

Most indexes of the NBTRC are structured and are classified into five relatively independent forms, including patient registration, admission record, operation record, discharge record and follow-up. The major data elements in each form are demonstrated in figure 2. 


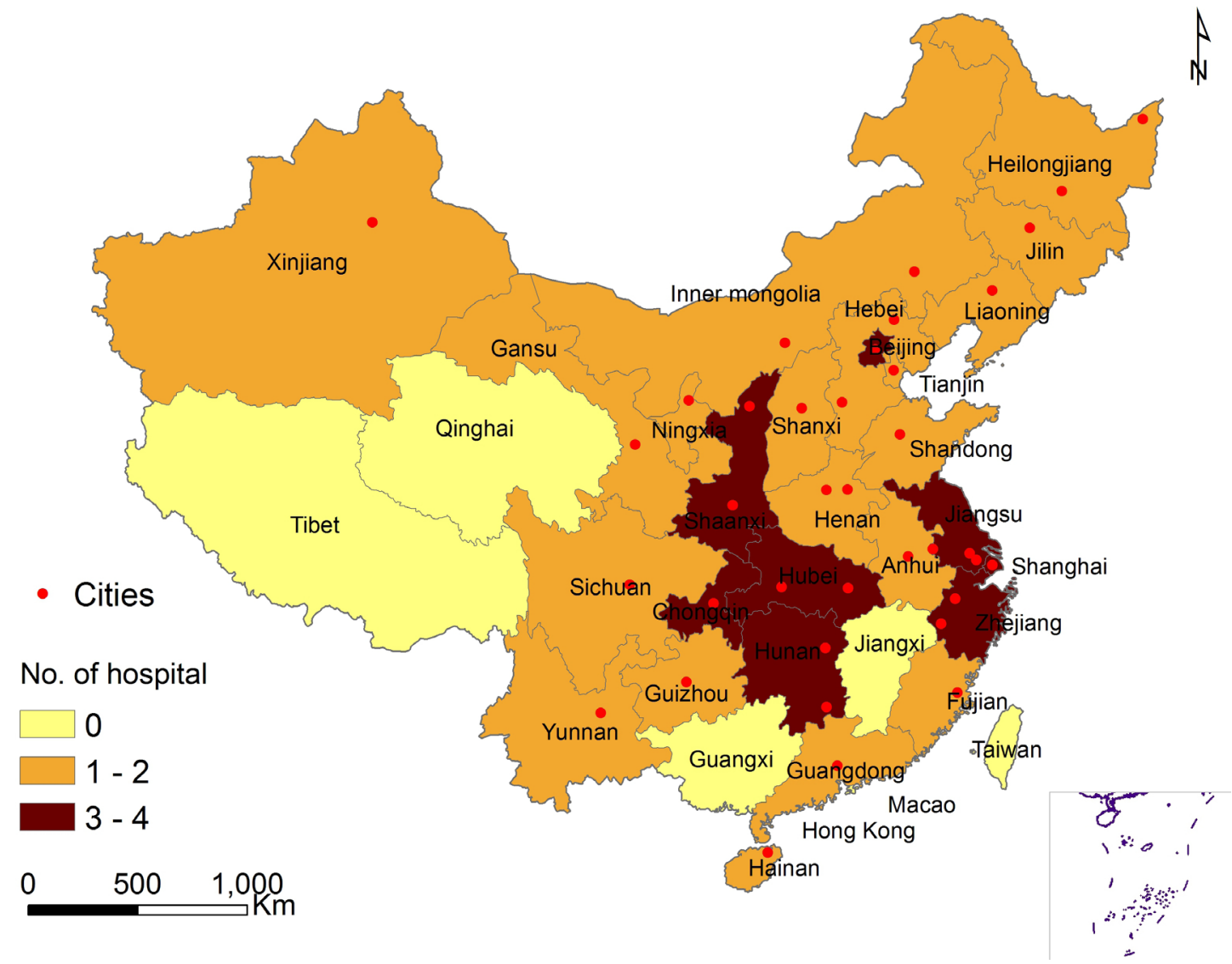

Figure 1 Spatial distribution of the participating hospitals of the National Brain Tumor Registry of China (NBTRC). As shown in the legend in the left lower part, the province/municipalities are colour-coded according to the cumulative number of participating hospitals of the NBTRC. The cities where the participating hospitals are located are indicated by red dots in China map.

\section{Data collection method}

The NBTRC has established three data upload interfaces for the participating hospitals, including the web portal and offline software (both requiring manual entry) and remote physical server that automatically abstracts data into the platform.

Based on the web portal interface, people can log in to the NBTRC web portal to fill in patients' information. The entered data are uploaded to the NBTRC database in real time via the internet.

While entering data via offline software, the data technician can use the offline software to fill in data and can upload data packages to the NBTRC database through the internet or by CD-ROM.

The remote physical server interface is the most convenient way to fill in data. The server is the middle layer between the NBTRC database and each hospital's information system, including unified medical record, surgical databases, radiology databases and administrative databases. With the authorisation of the data owner's hospital, the information system of that hospital will automatically upload data to the remote physical server on a regular basis. The software system in the remote physical server will extract and validate the data to each form of NBTRC and then send the normalised data to the platform through the internet or by CD-ROM.

\section{Functional modules of the NBTRC web portal system}

Six functional modules are designed within the established public web portal (figure 3). Each module is implemented in the web portal to accomplish various functions, as below.

The registration module is for the collection of in-hospital patient information and patient images. This module serves participating hospitals that upload data to the NBTRC online through the internet. Relevant web pages with different indicators are designed for generic and special brain tumour entities, according to different clinical features and indicators of interest. At present, the NBTRC sets up two special brain tumours-pituitary adenoma and brainstem tumour, which have different data entry web pages. Special brain tumours can be expanded according to certain clinical study in future.

The docking module interfaces the remote physical servers and the NBTRC database. The data can be uploaded to the platform through the remote physical servers in this module.

The follow-up module automatically provides the most appropriate follow-up questionnaire and time interval, 


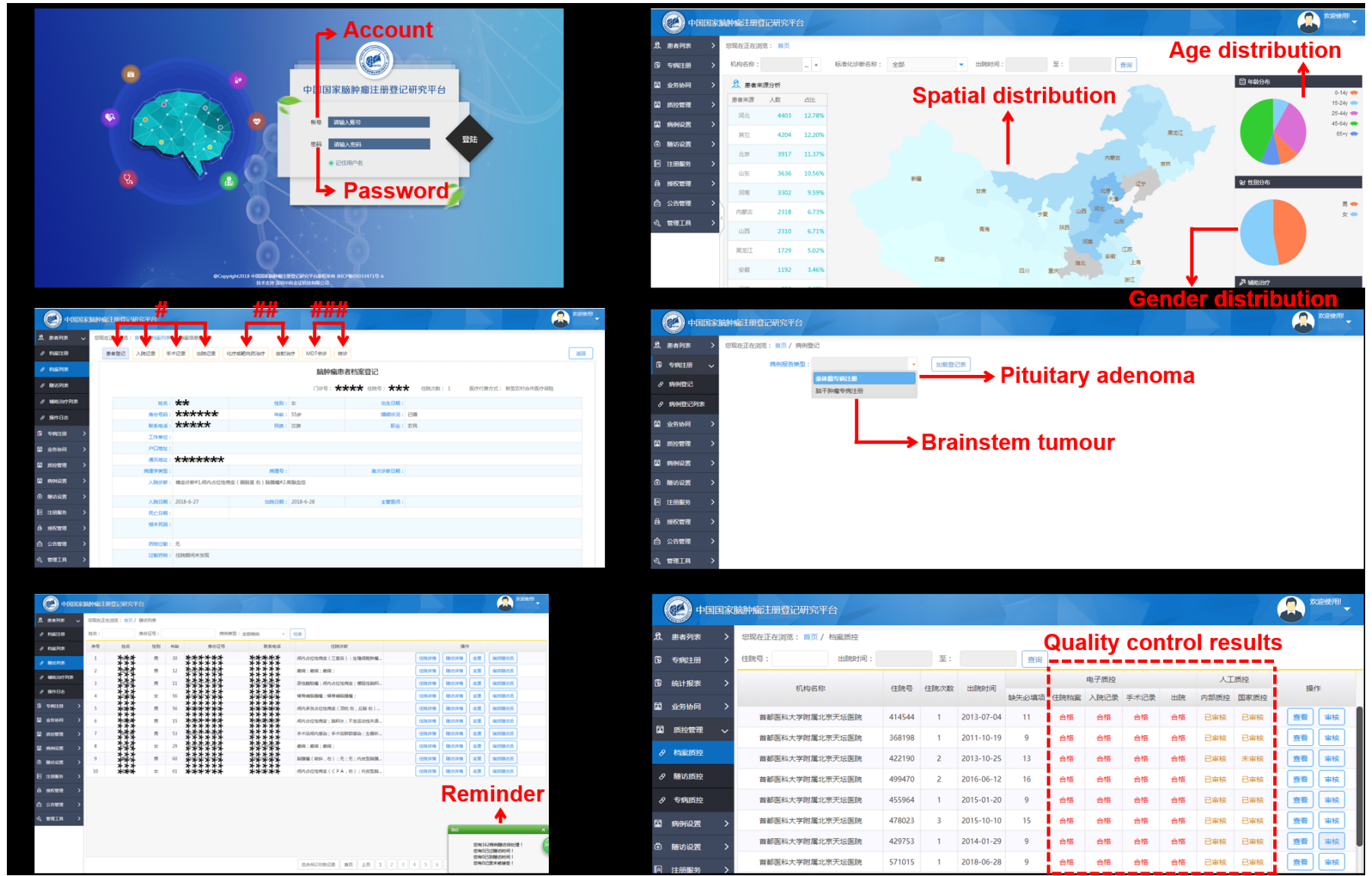

Figure 2 Components of the National Brain Tumor Registry of China web portal. (A) Login interface and (B) home page. This page shows the epidemiological distribution of patients with brain tumour and notifications. (C) Case report and cooperative medical service forms. \#Patients' in-hospital case report form including basic information and preoperative, intraoperative and postoperative recordings; \#\#case report form for patients receiving adjuvant therapy; \#\#\#cooperative medical service case report form including applications for referral and multidisciplinary team consults. (D) Case report form for patients with special brain tumour entities, including pituitary adenoma and brainstem tumour. (E) Patient follow-up data and questionnaire, with a flashing follow-up reminder. (F) Quality control results for each patient.

according to the pathology and location of the brain tumour. A friendly flashing reminder appears in the patient list when a follow-up time point is approaching.

The quality control module is used to analyse and display the quality of data from each participating hospital.

Through the cooperative medical service module, collaborative functions can be realised, such as patients' interhospital referrals and multidisciplinary consultations. In this system, we designed several function icons, including the application of further consultation or patient referral for the data entry surgeon, transferring the clinical data of interest to specific doctors and replying to the consultation comment or accepting the referral.

The authority management module sets permissions to upload and view the data and to the benefit-sharing roles of participating hospitals and end-users.

\section{Quality control}

The quality control of the NBTRC is the most important aspect of administration to ensure its scientific application. Therefore, the rigorous quality control indicators of each participating hospital's input data are carefully designed and maintained by the executive committee. Overall, seven quantifiable indicators are implemented: Without Interruption, Similar Monthly Volumes, Diagnostic Accuracy, Timeliness, Completeness, Percentage of Reported Cases and Follow-up Rate. The data quality will be an important reference for the benefits distributed to each participating hospital. The seven indicators and their thresholds are discussed below in detail.

First, Without Interruption means that the number of patients with brain tumour reported within any single month should not be zero. Similar Monthly Volume assumes that the number of reported patients within a single month should not vary by $20 \%$ more or $10 \%$ less from that of the same month in the previous 3 years. Diagnostic Accuracy means that the accuracy of pathological diagnoses of patients with brain tumour should be greater than $95 \%$, verified by the executive committee of the NBTRC through random selection and verification of at least $10 \%$ of specimens of patients with brain tumour. The fourth indicator, Timeliness, requires the 


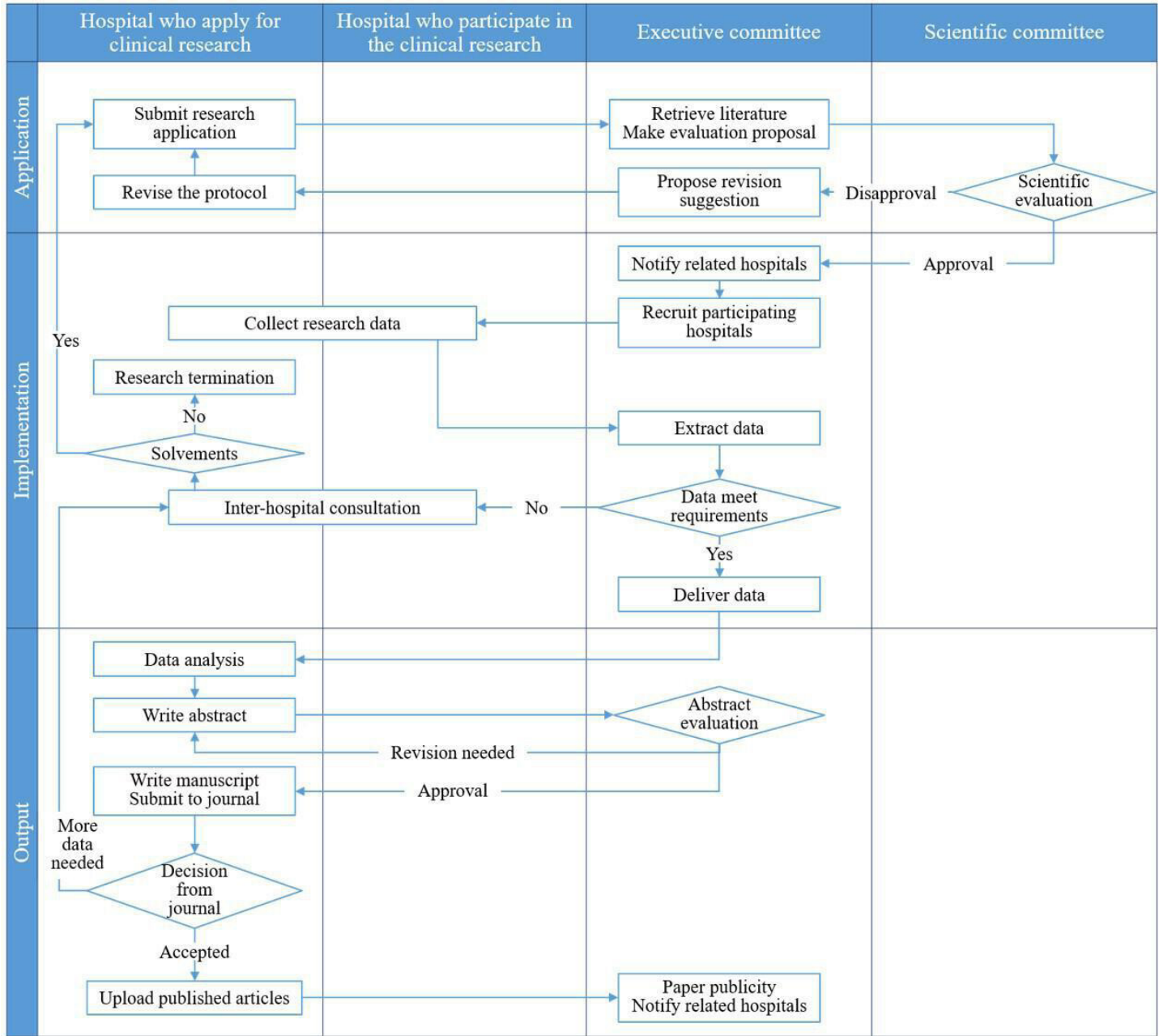

Figure 3 Schematic of workflow for conducting clinical research. The clinical research will be performed in an orderly manner in close cooperation with the participating hospitals of the National Brain Tumor Registry of China who apply for clinical research, contributing data hospitals, scientific committee and executive committee.

timely registry of patients' information within 5 days of hospital discharge. Completeness means that the completion rate of compulsory items of each patient should be $100 \%$. Percentage of Reported Cases requires that the number of patients with brain tumour reported by each participating hospital should be more than $95 \%$ of the total number of patients with brain tumour during the same interval. Finally, Follow-up Rate requires that the completion of at least one follow-up within 3 months after discharge should exceed $90 \%$.

\section{Information technology and data security of the NBTRC}

The hardware of the NBTRC is located in an independent and high-security data centre, which is operated by the NCRC-ND. The data centre includes dedicated physical server clusters, relational database system, Not Only Structured Query Language systems, object and block storages, intranet and networking devices, security hardware and software, and management utilities. For security, the data centre is not directly connected to the internet; instead, it provides the common data bus through the proxy server in the internet for external data traffic. In addition, the NBTRC implemented Secured
Online Application Programming Interfaces, based on which internet connection is allowed after strict authentication and authorisation.

\section{Benefit-sharing mechanism of the NBTRC}

The NBTRC allows all participating hospitals to share benefits based on their contributions.

There are dozens of pathological types of brain tumours according to the 2016 criteria of the WHO. ${ }^{27}$ The NBTRC selected several public subtypes of brain tumour that are of general interest in the field of neurosurgery. If the quantity and quality of the data from participating hospitals meet the pre-established standards, they can be applied for multicentre clinical research on the public subtypes of brain tumour.

In addition to the public subtypes of brain tumour, each participating hospital of NBTRC can select and apply a single subtype of brain tumour to conduct related clinical research. The scientific committee will assess the quantity and quality of the data from the hospital, as well as its clinical research capability. Only after the application is approved, that hospital acquires access to the multicentre data of the selected tumour subtype for clinical research. 


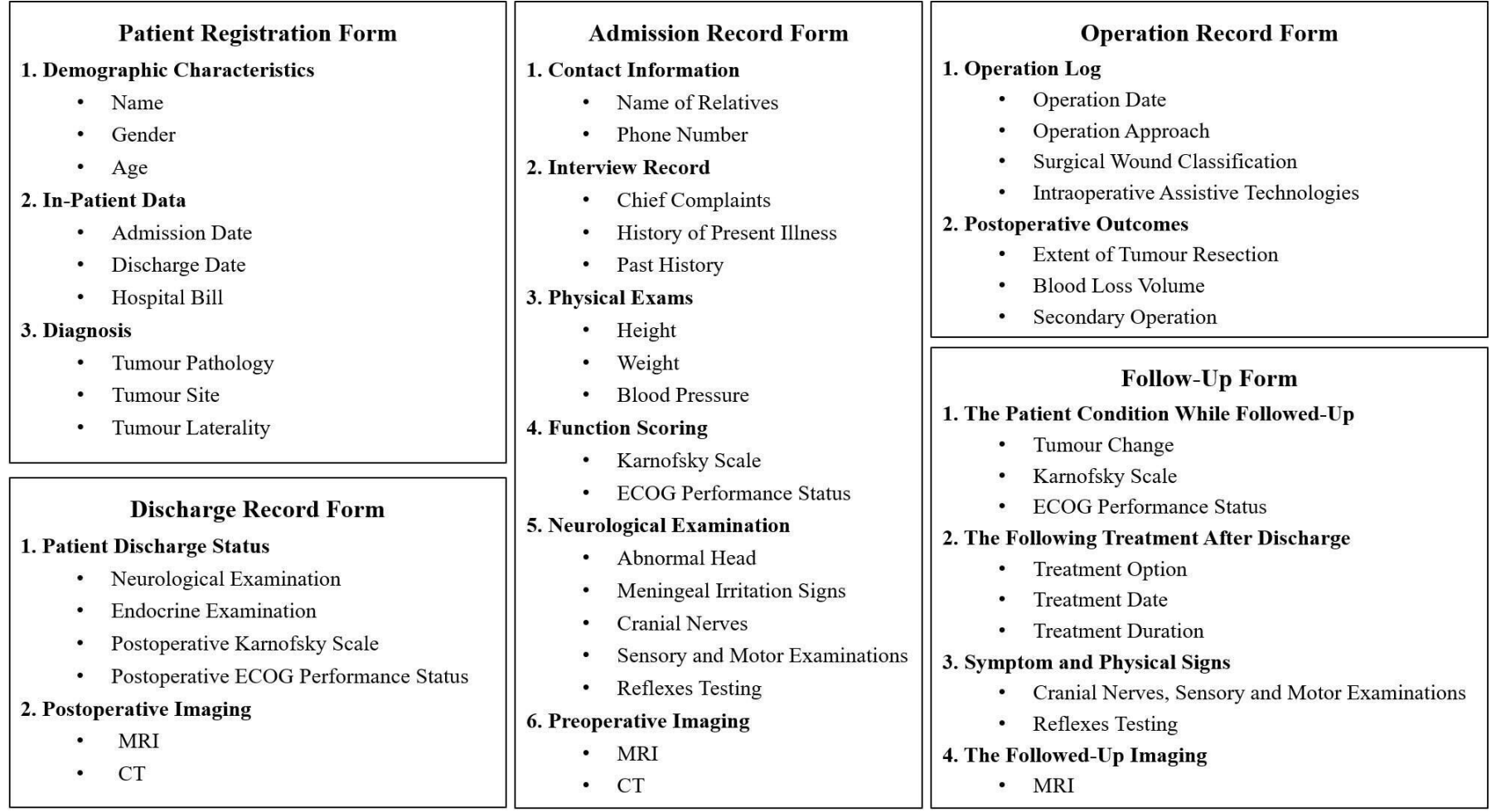

Figure 4 The major data elements in the five forms for data entry in the National Brain Tumor Registry of China. The five forms consist of patient registration form, discharge record form, admission record form, operation record form and follow-up form. There are many major data elements, respectively, in the five forms. ECOG, Eastern Cooperative Oncology Group.

The executive committee will periodically reassign the leader conducting related clinical research. The adjustment will consider the quantity and quality of clinical studies that have been conducted and the intention of each participating hospital.

The data-contributing hospital would share the achievements of the research such as papers, patents and scientific research awards, if the data that are uploaded to the platform had been used in a certain multicentre clinical research.

\section{Workflow of clinical research based on the benefit-sharing} mechanism

Each participating hospital is responsible for conducting clinical research that focuses on their allocated brain tumour subtype by using data from the NBTRC. A strict and standardised workflow is developed for conducting clinical research based on the data from the platform (figure 4).

First, the participating hospitals of the NBTRC fill out the standard research protocol form (table 1) and submit it to the scientific committee to apply for clinical research. The scientific committee evaluates the research protocol in terms of scientific and innovative quality and feasibility, and makes the decision. The executive committee assists in recruiting the hospitals willing to participate in the research and is responsible for organising and coordinating work among these hospitals. The researchers then collect and upload research data to the NBTRC. The executive committee extracts the data and delivers it to the principal investigator of this study. The researchers in the team of the principal investigator further analyse the data and write scientific reports. During this process, the executive committee would provide some assistance in statistics and edition, if needed. After publication of the report, the executive committee populates it on the NBTRC web portal and notifies the hospitals with data contribution.

\section{Patient and public involvement}

Patients or the public are not involved in the project.

Table 1 The research protocol form for all participating hospitals

\begin{tabular}{|c|c|}
\hline Name & \\
\hline Start ar & \\
\hline Resear & name of $\mathrm{PI}$ \\
\hline Particip & \\
\hline Particip & $n$ of work \\
\hline Backgr & \\
\hline Aim & \\
\hline Protocc & \\
\hline Time sc & \\
\hline Referer & \\
\hline Expect & \\
\hline & $\begin{array}{l}\text { Signature of the PI: } \\
\text { Date: }\end{array}$ \\
\hline
\end{tabular}




\section{DISCUSSION}

All parties using the NBTRC should benefit from the system based on their cooperation. Benefits to the government, participating hospitals and the patients with brain tumour are outlined below.

\section{Benefits of the government from the NBTRC}

By supporting the construction of the NBTRC, the government obtains reliable data to support public policy-making, such as the rational allocation of medical and health resource. The registration data will allow understanding of the status of brain tumours in Chinese population more comprehensively and accurately, including urgent needs for diagnosis, treatment and clinical research.

\section{Benefits of the participating hospitals from the NBTRC}

By taking part in the NBTRC, the participating hospitals could improve their capabilities of data management, clinical diagnosis and treatment, and scientific research. The standardised data and strict data quality control of NBTRC effectively resolve the common confusion on data management caused by inconsistent or missing data in the whole process of patient management including follow-up. In addition, after approval, each hospital could become the leader of national clinical research on a pathological subtype of brain tumour and use the data from all other participating hospitals. The multicentre and largesample studies have more possibility to influencing and innovative achievements on the national and global level. These hospitals will also benefit from participating in multicentre clinical studies that can improve their diagnosis and treatment capability and academic level, and promote their communication and cooperation.

\section{Benefits of the patients with brain tumour from the NBTRC}

Patients with brain tumour also obtain more high-quality, convenient and inexpensive medical services after being enrolled in the NBTRC. First, the unique referral system of the NBTRC facilitates the referral of patients to hospitals or medical teams that have higher diagnostic and treatment capabilities within the NBTRC system. If the data entry surgeon thinks that the patient is a difficult case with need for further consultation or treatment, they can apply for patient referral in the multicentre system. The necessary medical information can be transferred to the next doctor with demands of patient privacy protection. Consultation or accepting the patient referral can be replied in the system. In addition, the information networking mechanism of the NBTRC decreases the costs of repeated and redundant examinations and eliminates the inconvenience of patients carrying hardcopy radiological films between hospitals in different regions. ${ }^{28}$ Finally, the intelligent follow-up function of the NBTRC ensures that patients with brain tumour receive professional and timely medical consultation and assistance, which is conducive to doctor-patient communication, the improvement of patients' quality of life and better medical service.

In conclusion, the NBTRC is the first nation-wide data platform that consists of the entire spectrum of brain tumour entities in China. It is characterised by welldesigned organisation and management, unique benefitsharing mechanism and strict data quality control. As a model for better management and more efficient use of clinical data, the NBTRC provides a reliable reference for government's public policy-making process, helps healthcare professionals manage clinical brain tumour data, helps patients acquire high-quality and convenient medical services, and performs specific nation-wide multicentre clinical research.

\section{ETHICS AND DISSEMINATION}

This study received the ethical approval on 11 November 2019 and was registered in the Chinese Clinical Trial Registry. Informed consent will be obtained from all patients, and the confidentiality and anonymity of medical information will be fully guaranteed. Consent for the adolescents' participation will be also obtained from their parents or guardians via written consent. Data collection is being conducted continuously from 1 February 2019 to 31 January 2024. The results will be published in professional journals, in both Chinese and English.

Acknowledgements We are grateful to the Shenzhen United Imaging Healthcare Data Services $\mathrm{C}$. for the support in information technology.

Contributors LZ, NJ, DL, DX and G-LS helped with the conception and design. WJ and NJ conducted the platform supervision. DL, DX, TW and XX constructed the platform system. DX and DL wrote the manuscript. All authors approved the manuscript. All authors agree to be accountable for all aspects of the work.

Funding This work was sponsored by the National Key Technology Research and Development Program of the Ministry of Science and Technology of China (2013BAI09B03, 2014BAI04B01, 2015BAl12B04).

Map disclaimer The depiction of boundaries on the map(s) in this article does not imply the expression of any opinion whatsoever on the part of BMJ (or any member of its group) concerning the legal status of any country, territory, jurisdiction or area or of its authorities. The map(s) are provided without any warranty of any kind, either express or implied.

Competing interests None declared.

Patient and public involvement Patients and/or the public were not involved in the design, or conduct, or reporting, or dissemination plans of this research.

Patient consent for publication Not required.

Provenance and peer review Not commissioned; externally peer reviewed.

Open access This is an open access article distributed in accordance with the Creative Commons Attribution Non Commercial (CC BY-NC 4.0) license, which permits others to distribute, remix, adapt, build upon this work non-commercially, and license their derivative works on different terms, provided the original work is properly cited, appropriate credit is given, any changes made indicated, and the use is non-commercial. See: http://creativecommons.org/licenses/by-nc/4.0/.

ORCID iD

Deling Li http://orcid.org/0000-0003-1919-3765

\section{REFERENCES}

1 Kruchko C, Ostrom QT, Gittleman H, et al. The CBTRUS story: providing accurate population-based statistics on brain and 
other central nervous system tumors for everyone. Neuro Oncol 2018;20:295-8

2 Zeng $\mathrm{H}$, Chen W, Zheng R, et al. Changing cancer survival in China during 2003-15: a pooled analysis of 17 population-based cancer registries. Lancet Glob Health 2018;6:e555-67.

3 Lopes MBS. The 2017 World Health organization classification of tumors of the pituitary gland: a summary. Acta Neuropathol 2017; 134:521-35.

4 Collins FS, Varmus $\mathrm{H}$. A new initiative on precision medicine. N Engl J Med 2015;372:793-5.

5 Ostrom QT, Gittleman H, Liao P, et al. CBTRUS statistical report: primary brain and central nervous system tumors diagnosed in the United States in 2007-2011. Neuro Oncol 2014;16 Suppl 4:iv1-63.

6 Gunn ME, Malila N, Lähdesmäki T, et al. Late new morbidity in survivors of adolescent and young-adulthood brain tumors in Finland: a registry-based study. Neuro Oncol 2015;17:1412-8.

7 Narita Y, Shibui S, On Behalf of the Committee of Brain Tumor Registry of Japan Supported by the Japan Neurosurgical Society. Trends and outcomes in the treatment of gliomas based on data during 2001-2004 from the brain tumor registry of Japan. Neurol Med Chir 2015;55:286-95.

8 Wöhrer A, Waldhör T, Heinzl H, et al. The Austrian brain tumour Registry: a cooperative way to establish a population-based brain tumour Registry. J Neurooncol 2009;95:401-11.

9 Gittleman H, Ostrom QT, Farah PD, et al. Descriptive epidemiology of pituitary tumors in the United States, 2004-2009. J Neurosurg 2014;121:527-35.

10 Kshettry VR, Ostrom QT, Kruchko C, et al. Descriptive epidemiology of World Health organization grades II and III intracranial meningiomas in the United States. Neuro Oncol 2015;17:1166-73.

11 Villano JL, Parker CK, Dolecek TA. Descriptive epidemiology of ependymal tumours in the United States. Br J Cancer 2013;108:2367-71.

12 Gittleman HR, Ostrom QT, Rouse CD, et al. Trends in central nervous system tumor incidence relative to other common cancers in adults, adolescents, and children in the United States, 2000 to 2010. Cancer 2015;121:102-12.

13 Curran EK, Le GM, Sainani KL, et al. Do children and adults differ in survival from medulloblastoma? A study from the seer registry. $J$ Neurooncol 2009;95:81-5.

14 Curran EK, Sainani KL, Le GM, et al. Gender affects survival for medulloblastoma only in older children and adults: a study from the surveillance epidemiology and end results registry. Pediatr Blood Cancer 2009;52:60-4.

15 Ostrom QT, Cote DJ, Ascha M, et al. Adult glioma incidence and survival by race or ethnicity in the United States from 2000 to 2014 JAMA Oncol 2018;4:1254-62.

16 Deltour I, Johansen C, Auvinen A, et al. Time trends in brain tumor incidence rates in Denmark, Finland, Norway, and Sweden, 19742003. J Natl Cancer Inst 2009;101:1721-4.

17 Woehrer A, Lau CC, Prayer D, et al. Brain Tumor Epidemiology - A Hub within Multidisciplinary Neuro-oncology. Report on the 15th Brain Tumor Epidemiology Consortium (BTEC) Annual Meeting, Vienna, 2014. Clin Neuropathol 2015;34:40-6.

18 Arora RS, Alston RD, Eden TOB, et al. Are reported increases in incidence of primary CNS tumours real? an analysis of longitudinal trends in England, 1979-2003. Eur J Cancer 2010;46:1607-16.

19 Lym RL, Ostrom QT, Kruchko C, et al. Completeness and concordancy of WHO grade assignment for brain and central nervous system tumors in the United States, 2004-2011. J Neurooncol 2015;123:43-51.

20 Chen W, Zheng R, Zeng $\mathrm{H}$, et al. Annual report on status of cancer in China, 2011. Chin J Cancer Res 2015;27:2-12.

21 Chen W, Zheng R, Zeng $\mathrm{H}$, et al. The updated incidences and mortalities of major cancers in China, 2011. Chin J Cancer 2015;34:502-7.

22 Chen W, Zheng R, Baade PD, et al. Cancer statistics in China, 2015. CA Cancer J Clin 2016;66:115-32.

23 Jiang T, Tang G-fu, Lin Y, et al. Prevalence estimates for primary brain tumors in China: a multi-center cross-sectional study. Chin Med J 2011;124:2578-83.

24 Yang J, Siri JG, Remais JV, et al. The Tsinghua-Lancet Commission on healthy cities in China: unlocking the power of cities for a healthy China. The Lancet 2018;391:2140-84.

25 Tang J, Chen C, Zhao Y. Neurosurgery center of Beijing Tiantan Hospital, flagship of neurosurgery in China. World Neurosurg 2011;75:377-82.

26 Jacobson RS, Becich MJ, Bollag RJ, et al. A Federated network for translational cancer research using clinical data and biospecimens. Cancer Res 2015;75:5194-201.

27 Louis DN, Perry A, Reifenberger G, et al. The 2016 World Health organization classification of tumors of the central nervous system: a summary. Acta Neuropathol 2016;131:803-20.

$28 \mathrm{Li} \mathrm{X}, \mathrm{Lu} \mathrm{J}, \mathrm{Hu}$ S, et al. The primary health-care system in China. Lancet 2017;390:2584-94. 